D.T. Wickramasinghe, L. Ferrario, and G.V. Bicknell, eds.

\title{
Self-Similar MHD Collimated Outflows
}

\author{
E. Trussoni \\ Osservatorio Astronomico di Torino, Pino Torinese, Italy \\ C. Sauty \\ Observatoire de Paris, DAEC, Meudon, France \\ K. Tsinganos \\ University of Crete, Dept. of Physics, Heraklion, Greece
}

\begin{abstract}
We present a class of steady axisymmetric MHD solutions, self-similar in the meridional direction, that are suitable to model collimated winds accelerated in the potential well of a spherical object.
\end{abstract}

The approach of self-similarity is often followed to model astrophysical outflows as a way to overcome the mathematical complexity of the axisymmetric MHD equations. In particular, self-similar solutions in the meridional direction allow to analyze the properties of a wind emerging from a magnetized and rotating spherical gravitating object close to its polar axis. In a spherical frame of reference we make the following two basic assumptions (Trussoni et al. 1996): (i) the magnetic flux function, and thence the poloidal components of the velocity and magnetic field, are factorized: $A(R, \theta) \propto f(R) \sin ^{2} \theta$; (ii) the density and the gas pressure scale linearly with $A: \rho(R, \theta) \propto 1+\delta A$ and $P(R, \theta) \propto P_{o}(R)+P_{1}(R) A / f$. Then, for spherical poloidal Alfvén surfaces, $M(R, \theta) \equiv M(R)$, the MHD equations reduce to a system of three ordinary differential equations for $M, f, P_{o}$ and $P_{1}$. To close this system, we can either prescribe 'a priori' the shape of the poloidal streamlines, $f(R)$, or relate the pressure components as $P_{1} \propto P_{o}$ and deducing the streamline pattern $f(R)$.

Here we shortly discuss the case of winds with prescribed streamlines such that they are collimated far from the central body (for the other class of solutions, see Trussoni et al. 1996). For this purpose we assume $f(R) \propto 1+\left(R / R_{c}\right)^{2}$, where $R$ is the radial coordinate in units of the radius of the Alfvén surface, and $R_{c}$ is the collimation distance. If $R_{c} \gg 1$ the streamlines are radial in the subAlfvénic and transAlfvénic region, and cylindrically parallel to the rotational axis asymptotically. By imposing force balance across the streamlines for $R \gg R_{c}$, we find that the wind always attains superAlfvénic terminal velocities $\left(M_{\infty} \equiv\right.$ const. $\left.\gg 1\right)$ if the flow is overpressured on its axis [i.e. $q<0$, where $q \propto\left(P_{1} / R^{2}\right)_{\infty} \equiv$ const]. In such a case the pinching of the external toroidal magnetic field always balances the sum of the centrifugal force and thermal pressure gradient. If on the other hand the wind is underpressured $(q>0)$, the dynamics is more complex: two branches of solutions exist, depending on whether the centrifugal force is balanced by the toroidal magnetic field (magnetic regime) or 

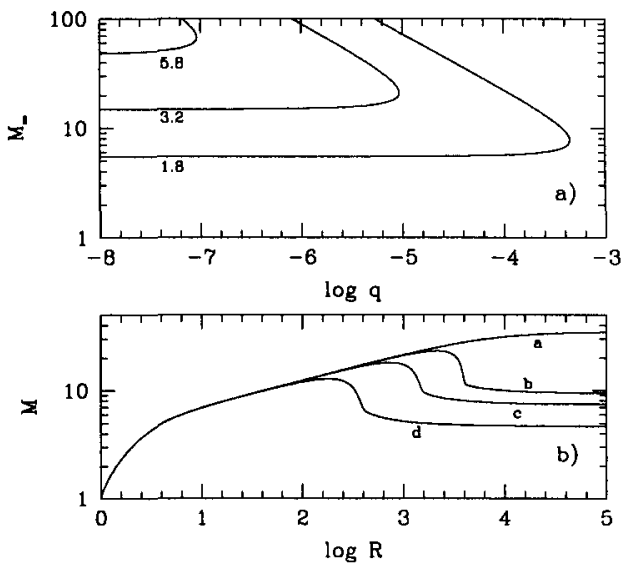

Figure 1. Panel a: $M_{\infty} v s . q$ for $q>0$ and some values of $R_{c}$. The thermal and magnetic regimes are on the upper and lower branches, respectively. Panel b: $M$ vs $R$ for $R_{c}=3.3$ and $q=5.6 \times 10^{-4}$ (a), $-1.4 \times 10^{-4}(\mathrm{~b}),-4.6 \times 10^{-4}(\mathrm{c})$ and $-3.7 \times 10^{-3}(\mathrm{~d})$. Only the solution with $M$ monotonically increasing is in the thermal regime for $R \gg 1$.

by the pressure gradient (thermal regime). Furthermore, there is a minimum value for the pressure on the axis (i.e. a maximum value of $q$ ) below which the inwards pinching prevails, hampering any collimated equilibrium (see Fig. 1a).

The complete solutions connecting the subAlfvénic to the superAlfvénic regions, smoothly crossing the Alfvén surface, require the numerical integration of the full MHD system. It is found that two classes of solutions exist, depending on the value of the asymptotic pressure on the rotational axis. If it vanishes, then $M(R)$ increases monotonically, while if it is finite asymptotically, the Alfvén number has maximum in the intermediate region. In this last case the flow is always overpressured $(q<0)$ and magnetically confined. In the former case the wind is underpressured $(q>0)$ and is magnetically or thermically confined depending on whether the rotational speed is fast or slow (see Fig. 1b, for slow rotation).

We conclude by remarking that the present meridionally self-similar treatment can be considered complementary to the radially self-similar one which is widely followed to model winds from accretion disks (Blandford \& Payne 1982).

\section{References}

Blandford, R.D \& Payne, D.G. 1982, MNRAS, 199, 883

Trussoni, E., Sauty, C. \& Tsinganos, K. 1996, in Solar and Astrophysical MHD Flows, K. Tsinganos (ed.), Kluwer Academic Publishers, 383 